\title{
Clarification des fondements épistémologiques de la recherche fondamentale à visée pragmatique concernant le fonctionnement humain optimal
} lever quelques ambiguïtés, controverses et/ou polémiques suscitées par la psychologie positive

Clarifying epistemological foundations of fundamental research with pragmatic focus on the optimal human functioning. Clear some ambiguities, controversies and/or polemics generated by the positive psychology

\section{Jean Heutte}

\section{OpenEdition}

\section{Journals}

Édition électronique

URL : https://journals.openedition.org/trema/5611

DOI : $10.4000 /$ trema.5611

ISSN : 2107-0997

Éditeur

Faculté d'Éducation de l'université de Montpellier

Référence électronique

Jean Heutte, «Clarification des fondements épistémologiques de la recherche fondamentale à visée pragmatique concernant le fonctionnement humain optimal », Tréma [En ligne], 52 | 2019, mis en ligne le 01 septembre 2019, consulté le 16 janvier 2023. URL : http://journals.openedition.org/trema/5611 ; DOI : https://doi.org/10.4000/trema.5611

Ce document a été généré automatiquement le 16 janvier 2023.

Tous droits réservés 


\section{Clarification des fondements} épistémologiques de la recherche fondamentale à visée pragmatique concernant le fonctionnement humain optimal

lever quelques ambiguïtés, controverses et/ou polémiques suscitées par la psychologie positive

Clarifying epistemological foundations of fundamental research with pragmatic focus on the optimal human functioning. Clear some ambiguities, controversies and/or polemics generated by the positive psychology

\section{Jean Heutte}

L'auteur remercie le programme Interreg Dig-e-Lab, pour le soutien du projet qui a permis de formaliser la réflexion présentée dans cet article, via le Fond européen de développement régional (FEDER). Met steun van het Europees Fonds voor Regionale Ontwikkeling.

En premier lieu, cet article souhaite retracer rapidement les fondements épistémologiques (historique, philosophique, méthodologique...) de la science de l'expérience optimale en tant que champ de recherche empirique concernant l'expérience positive subjective (Brown, Lomas \& Eiroa-Orosa, 2017), depuis la création du Positive Psychology Steering Commitee constitué par Mihaly Csikszentmihalyi, Ed Diener, Kathleen Hall Jamieson, Chris Peterson et George Vaillant, en 1999, à Akumal (Mexique), jusque ses dernières évolutions notamment sous l'influence des chercheurs européens. Il a aussi pour objectif de lever quelques ambiguïtés originelles concernant certains termes (optimal, positif et positiviste) qui parfois peuvent être l'objet de controverses (voire de polémiques idéologiques infondées) quand ils sont mal traduits ou interprétés. Bien entendu, comme de nombreux scientifiques, nous souhaitons affirmer haut et fort que nous ne soutenons aucune dérives, qu'elles soient mercantiles 
ou éthiquement douteuses, de la part de celles et ceux qui revendiquent s'inscrire dans le champ de la psychologie positive ou agir en son nom. Cependant, force est de constater que certains discours à charge contre la psychologie positive relèvent de procès d'intention en amalgamant souvent des griefs qui ne sont pas nécessairement infondés (et que nous pouvons parfois tout à fait partager), mais qui ne concernent en rien la psychologie positive. Si ces auteurs sont de bonne foi, c'est probablement qu'ils sont dans l'ignorance de leur ignorance et qu'ils sont de ce fait dans l'incapacité de dépasser le pic de stupidité (effet "Dunning-Kruger"1). Dans le cas contraire, c'est qu'ils se situent sur un terrain qui n'est pas celui de la controverse scientifique, mais dans une polémique idéologique qui mérite d'être remise à sa juste place: c'est indirectement à cela que nous souhaitons aussi contribuer.

\section{L'émergence de la préoccupation du développement humain optimal}

2 Pendant longtemps, la psychologie conventionnelle s'est plutôt intéressée aux aspects négatifs de la vie humaine. Il y a bien eu quelques îlots d'intérêt pour des sujets tels que la créativité, l'optimisme et la sagesse, mais ils n'ont pas été unis derrière une grande théorie ou un cadre général. Cette situation plutôt négative n'était pas l'intention originelle des premiers psychologues, mais plutôt le résultat d'un accident historique.

3 En effet, des points de vue qu'il est possible de qualifier rétrospectivement d'humanistes remontent aux origines modernes de la psychologie (Shaffer, 1978). James (1890), en particulier, a fait valoir que pour étudier en profondeur le fonctionnement humain optimal, il faut tenir compte de l'expérience subjective d'un individu. Pour cette croyance, et d'autres, James est considéré par certains comme «le premier psychologue positif de l'Amérique » (Taylor, 2001, p. 15, traduction personnelle). Selon Rathunde (2001), les approches expérientielles de James, Dewey et Maslow sont conformes aux philosophies du pragmatisme (Peirce, 1877; Peirce \& Hetzel, 1878), de l'existentialisme (Kierkegaard, 1843; Heidegger, 1927; Sartre, 1943) et de la phénoménologie (Husserl, 1913/1950). Chacune d'entre elles fait référence à « l'intense subjectivité » de l'expérience immédiate (Rathunde, 2001) et constitue donc un défi de taille pour les conceptions traditionnelles de la méthode scientifique. Bien qu'aucun d'entre eux ne rejette l'importance de la rationalité et des modes d'analyse rigoureux et objectifs (c'est-à-dire les qualités cognitives qui sont généralement considérées comme étant les plus représentatives de la science), ils ne glorifient pas ces outils cognitifs ou ne les placent pas au-dessus et au-delà de la nature humaine. Au lieu de cela, ils soutiennent que l'objectivité est plus souvent conçue comme un processus rationnel permettant de parvenir à un consensus dans un lieu et à un moment précis.

4 Cette combinaison de méthodologie positiviste et phénoménologique était connue sous le nom d'« empirisme radical ». Non seulement James s'intéressait à ce qui était objectif et observable, mais aussi à ce qui était subjectif parce que « l'objectivité est basée sur une subjectivité intense » (Gilkey, 1990, cité dans Rathunde, 2001, p. 139, traduction personnelle).

5 Dans son allocution présidentielle à l'occasion du congrès annuel de l'American Psychological Association, le 28 décembre 1904, à Philadelphie, James se demandait pourquoi certaines personnes étaient capables d'utiliser leurs ressources à leur pleine 
capacité et d'autres pas. Pour comprendre cela, selon lui, il fallait répondre à deux autres questions : « (a) quelles sont les limites de l'énergie humaine? et (b) comment cette énergie pourrait-elle être stimulée et libérée pour être utilisée de façon optimale?» (Rathunde, 2001, p. 142, traduction personnelle). Ces questions sont une démonstration claire de l'expérience de James, notamment de son intérêt pour l'étude $\mathrm{du}$ fonctionnement optimal de l'être humain et son rapport à l'expérience, un fil conducteur tissé en commun dans toute la littérature de psychologie positive.

6 Ainsi, avant la Seconde Guerre mondiale, la psychologie avait trois tâches : (1) guérir la maladie mentale, (2) améliorer la vie normale et (3) identifier et cultiver les grands talents. Cependant, après la guerre, les deux dernières tâches se sont perdues, concentrant principalement les travaux sur la première. Cela était lié d'une part à la réduction brutale des financements par les organismes gouvernementaux (en raison des priorités de la reconstruction), et d'autre part au besoin de faire face à une crise psychique de très grande ampleur (notamment concernant les vétérans), toutes les ressources disponibles ont été consacrées à l'apprentissage et au traitement des troubles psychologiques et à la psychopathologie. Bien entendu, cela s'est fortement ressenti au niveau des publications scientifiques. Ainsi, suite à une recherche via la base de données Psycinfo, sur la période entre 1967 et 2000, Myers (2000) constate que 5548 articles ont été publiés sur la colère, 41416 sur l'anxiété et 54040 sur la dépression, mais seulement 415 sur la joie, 1710 sur le bonheur et 2582 sur la satisfaction à l'égard de l'existence. Le rapport est de 21 articles sur les émotions négatives pour un article sur les émotions positives.

7 Sur la durée, ce déséquilibre n'a pas été sans conséquence sur les modèles théoriques élaborés par les chercheurs. La concentration de la production scientifique sur les troubles psychiques et les maladies mentales, pendant plusieurs décennies, a ainsi progressivement $\mathrm{pu}$ introduire quelques biais dans certaines interprétations de données scientifiques, sans que personne n'y prête réellement garde, comme l'illustre ici Jacques Lecomte :

Un exemple est remarquable à cet égard (Lecomte, 2002). La plupart des enfants maltraités deviennent des parents affectueux (80-90\% dans la majorité des études). Beaucoup de personnes pensent le contraire, l'illusion cognitive venant du fait que la plupart des parents maltraitants ont été maltraités. Et l'on glisse, sans s'en rendre compte, d'une démarche rétrospective à une démarche prospective. [...] Il est impressionnant de constater que pratiquement toutes les études sur ce thème utilisent l'expression «transmission (ou reproduction) intergénérationnelle de la maltraitance ». Or, il est rhétoriquement très diffèrent de dire qu'il y a une faible transmission intergénérationnelle de la maltraitance ou de dire qu'il y a une forte cessation intergénérationnelle de la maltraitance, même si les deux formules sont objectivement, scientifiquement exactes. Dans le premier cas de figure, le propos se situe dans le paradigme de la reproduction de la violence, et donc d'une vision négative de l'être humain. Ceci relève clairement d'un rapport aux valeurs (selon l'expression de Max Weber), même inconscient. (Lecomte, 2012, p. 24).

8 Afin de pouvoir mieux situer l'émergence du courant de la psychologie positive, il semble intéressant de revisiter les vagues précédentes qui ont marqué son histoire. 


\section{Les quatre vagues de l'histoire de la psychologie}

9 La psychologie positive peut être considérée comme la quatrième vague dans l'évolution de la psychologie, les trois premières vagues étant, respectivement, le modèle de la maladie, le behaviorisme et la psychologie humaniste.

\section{La première vague : le modèle de la maladie}

En faisant de l'inconscient le concept majeur de compréhension de l'esprit humain, Sigmund Freud soutient que les raisons du comportement échappent le plus souvent à la conscience. Cette idée va révolutionner les pratiques thérapeutiques. De ce fait, pendant la seconde moitié du XIXe siècle et au début du XXe siècle, la psychologie se préoccupe principalement de guérir les troubles mentaux. Suite aux publications de Freud, Adler et Jung, ces thérapies sont très influencées par la psychanalyse. Par la suite, notamment via l'influence des travaux d'Ellis, Beck, Bandura, Seligman..., ce sont les thérapies cognitives comportementales (TCC) qui se sont diffusées parmi les praticiens.

11 Le modèle de maladie s'est avéré très utile. Seligman souligne les victoires de ce modèle, qui sont, par exemple, que quatorze maladies mentales auparavant incurables peuvent maintenant être traitées avec succès. Cependant, le coût de l'adoption de ce modèle a entraîné trois inconvénients majeurs particulièrement négatifs pour cette communauté de chercheurs et de praticiens: (1) les psychologues sont devenus des victimologues et des pathologisateurs, (2) ils ont oublié d'améliorer la qualité de vie normale, ainsi que de contribuer à l'identification et l'épanouissement des personnes talentueuses et (3) dans leur hâte de réparer les dommages, il ne leur est jamais venu à l'idée de développer des interventions pour rendre les gens plus heureux (Seligman \& Csikszentmihalyi, 2000).

\section{La deuxième vague : le behaviorisme}

12 Skinner, de l'Université de Harvard, fut l'initiateur (avec Watson et Pavlov) de l'approche comportementale en psychologie. Skinner pensait que le libre arbitre était une illusion et que le comportement humain dépendait largement de la compréhension des conséquences de nos actions précédentes. En totale opposition à la psychanalyse, il rejette la méthode introspective, lui reprochant de ne s'appuyer que sur le subjectif. Il considère que les scientifiques ne doivent étudier les phénomènes qu'à partir de faits observables, ce qui va fortement réduire le champ de la psychologie scientifique. Suite à de très nombreuses expériences, il développe le concept de conditionnement opérant, notamment via des renforcements positifs (vs renforcements négatifs).

Il est à noter qu'à l'époque, Skinner (1971) a sérieusement critiqué l'enseignement traditionnel fondé essentiellement sur des renforcements négatifs et a proposé de remplacer ceux-ci par des renforcements positifs. En cela, il pourrait presque être considéré comme l'un des pionniers de l'éducation positive (Heutte, 2019). A ceci près que son opposition farouche aux sciences cognitives et sa vision très mécanique ( $c f$. Teaching machines, inspirées des travaux de Pressey (1927)) de l'apprentissage (Skinner, $1958,1981,1984)$ a parfois fortement brouillé son message auprès de la Commission on Behavioral and Social Sciences and Education du National Research Council (1984). Pour sa 
part, Csikszentmihalyi déplore surtout que progressivement, notamment à l'apogée du behaviorisme, la psychologie ait été enseignée comme s'il s'agissait d'une branche de la mécanique statistique et surtout qu'elle ait été incapable de «concilier les impératifs jumeaux qu'une science du comportement humain doit inclure : comprendre ce qui est et ce qui pourrait être» (Seligman \& Csikszentmihalyi, 2000, p. 7, traduction personnelle).

\section{La troisième vague : la psychologie humaniste}

La psychologie humaniste s'est développée vers le milieu du vingtième siècle en partie pour proposer une alternative ( $c f$. the "third way") à la psychanalyse et au behaviorisme: ces deux branches principales de la psychologie semblaient avoir apporté de grandes contributions à la connaissance humaine, mais ni isolément, ni ensemble, elles ne réussissaient réellement à couvrir l'étendue presque illimitée du comportement, des relations et des possibilités humaines. D'autre part, à cette époque, Maslow constate que "la psychologie a connu beaucoup plus de succès sur le plan négatif que sur le plan positif : elle nous a beaucoup appris sur les défauts de l'homme, sa maladie, ses péchés, mais peu sur ses potentialités, ses vertus, ses aspirations réalisables ou ses ressources psychiques. C'est comme si la psychologie s'était volontairement limitée à la moitié de sa compétence légitime, et de plus à la moitié la plus sombre et la plus mauvaise» (1954, cité par Froh, 2004, p. 19, traduction personnelle). Selon Sutich et Vich (1969), la plus grande limitation de la psychanalyse et du behaviorisme est l'insuffisance de leur approche des potentialités humaines positives et la réalisation maximale de ces potentialités.

Ainsi, même si dès le départ, la psychologie positive a souhaité prendre des distances avec la psychologie humaniste, il est difficile de nier que la psychologie humaniste en est une source d'inspiration. En effet, tout d'abord, il est important de reconnaître que la paternité du terme Positive Psychology revient à Maslow dont le dernier chapitre du livre Motivation and Personality publié en 1954 s'intitule Toward a Positive Psychology, chapitre dans lequel il appelait à porter une plus grande attention aux aspects positifs (vs négatifs) de l'expérience humaine. Enfin, poursuivant la voie ouverte par James en son temps, Maslow et Rogers s'intéressent tour les deux au fonctionnement optimal de l'être humain, même si leurs conceptions n'en représentent pas réellement les mêmes points de vue :

- Pour Maslow il s'agit plutôt d'un processus d'actualisation permanent, lié à un trait de personnalité: le fonctionnement optimal correspond à «un processus continuel de réalisation de leurs potentiels, capacités et talents, ce qui apparaît comme l'accomplissement d'une mission (qu'elle soit appelée destinée ou vocation), comme un savoir accru et une acceptation de la nature intrinsèque d'une personne, comme une tendance incessante vers l'unité, l'intégration ou la synergie à l'intérieur d'un individu ». (1968, cité par Waterman, 1984, p. 332, traduction personnelle)

- Pour sa part, Rogers décrit le fonctionnement optimal de la personnalité comme étant plutôt le fruit d'un changement d'état lié à une transformation : le passage « d'un état de rigidité à un état de flexibilité, d'une vie statique à une vie active, de la dépendance à l'autonomie, d'un état de vie prévisible à celui de créativité imprévisible et d'une attitude de défense à une attitude d'acceptation de soi ». (1973, p. 14, traduction personnelle) 
Csikszentmihalyi (Seligman \& Csikszentmihalyi, 2000) reconnaît que la vision généreuse liée à la "troisième voie" annoncée par Maslow, Rogers et d'autres psychologues humanistes a eu un fort impact sur la culture en général et était très prometteuse. Cependant, il déplore la faiblesse de sa base empirique cumulative : la psychologie humaniste a plutôt engendré une myriade de mouvements d'autoassistance thérapeutique qui parfois mettaient l'accent sur le soi et encourageaient l'égocentrisme, tout en minimisant les préoccupations pour le bien-être collectif. Seligman et Csikszentmihalyi (2000) laissent au débat futur de déterminer si c'est parce que Maslow et Rogers étaient en avance sur leur temps, parce que ces défauts étaient inhérents à leur vision originale, ou à cause de "suivants" trop enthousiastes. Mais ils constatent que l'un des héritages des années 1960 est clairement en évidence dans n'importe quelle grande librairie : «la section "psychologie" contiendra au moins dix étagères sur la guérison par le cristal, l'aromathérapie ou comment atteindre l'enfant qui est en nous, pour chaque étagère de livres qui tente de rester conforme à une certaine norme scientifique» (Seligman \& Csikszentmihalyi, 2000, p. 7, traduction personnelle).

Ainsi, il est certainement judicieux de conclure provisoirement que si dans un premier temps afin de marquer son territoire, la psychologie positive a opportunément souhaité se démarquer de la psychologie humaniste, c'est plutôt pour des soucis de formes (de méthodes) que de fond.

\section{La quatrième vague : la psychologie positive}

Il est désormais admis que l'article co-signé par Seligman et Csikszentmihalyi (2000) dans le premier $\mathrm{n}^{\circ} \mathrm{du}$ millénaire de la revue American Psychologist constitue symboliquement l'acte scientifique fondateur majeur de la psychologie positive.

19 Au-delà des origines personnelles de leurs convictions, par l'écriture conjointe de l'article séminal, ils annoncent que le temps est arrivé de fonder scientifiquement la psychologie positive.

Dans cet article, ils rappellent que :

- Le champ de légitimité de la psychologie n'est pas seulement l'étude de la pathologie, de la faiblesse et des dommages; c'est aussi l'étude de la force et de la vertu.

- Le traitement n'est pas seulement de réparer ce qui est cassé ; c'est de favoriser ce qu'il y a de mieux.

- La psychologie n'est pas seulement une branche de la médecine qui s'intéresse à la maladie ou à la santé ; elle s'intéresse à un domaine beaucoup plus vaste, notamment: le travail, l'éducation, la perspicacité, l'amour, la croissance, le jeu...

21 Et dans cette quête de ce qu'il y a de mieux, « la psychologie positive ne s'appuie pas sur des vœux pieux, la foi, l'illusion de soi, les modes ou l'agitation des bras; elle essaie d'adapter les meilleures méthodes scientifiques aux problèmes uniques que présente le comportement humain à ceux qui veulent le comprendre dans toute sa complexité " (Seligman et Csikszentmihalyi, 2000, p. 7, traduction personnelle). 


\section{Lever trois ambiguïtés originelles : optimal, positif et positiviste} d'interrogation, de controverses et/ou parfois même de polémiques, voire de procès d'intention. Quoi qu'il en soit, afin de rendre plus compréhensible notre propos, autant lever tout de suite toutes les ambiguïtés possibles.

\section{Être ou ne pas être optimal ?}

La psychologie positive se définit comme «la science qui étudie le fonctionnement humain optimal », c'est-à-dire qu'elle vise à isoler et à mettre en valeur les variables favorables à l'épanouissement des individus et des communautés.

Ainsi, le terme « optimal » ne fait en aucun cas référence à une forme d'optimalisation ou d'optimisation :

- aucun lien avec les démarches consistant à rendre optimal le fonctionnement d'un système. L'optimalisation c'est la recherche de l'efficacité, le gain de temps et de productivité. Le Taylorisme, qui instaure le travail à la chaîne, la robotisation, l'informatisation, sont des optimalisations.

- aucun lien avec l'optimisation combinatoire (qui consiste à trouver dans un ensemble discret un parmi les meilleurs sous-ensembles (ou solutions) réalisables) qui est liée aux champs théoriques de la combinatoire, des mathématiques discrètes ou de la théorie des graphes (une branche des mathématiques née au vingtième siècle pour répondre aux besoins de divers domaines: l'informatique, l'organisation de la production à grande échelle, la gestion des opérations militaires, l'économie...)

- aucun lien avec l'optimisation du code informatique liée au souhait d'améliorer un programme ou une librairie logicielle,

- aucun lien avec l'optimisation pour l'analyse économique et les sciences de gestion,

- aucun lien avec l'optimalisation ou optimisation fiscale

$\cdot \ldots$

Pour être plus clair encore, le fonctionnement psychologique optimal ne fait pas référence à une performance liée à un jugement social ou une norme externe au sujet. Cela peut cependant éventuellement faire référence à une performance auto-référée, liée à un défi personnel (Martin-Krumm \& Tarquinio, 2011) : un niveau de réalisation (ou d'exigence) qui va permettre l'épanouissement de la personne, en opposition totale avec l'idée de la compétition, du record ou de la performance tel que cela peut se comprendre dans un sens commun.

Comme indiqué précédemment, historiquement, la conception de James (1906, cité par Rathunde, 2001) sous-tend que le fonctionnement optimal est celui qui permet à la personne de donner le meilleur d'elle-même, d'être capable d'utiliser ses ressources à leur pleine capacité. Pour Maslow (1968), il s'agit plutôt d'un processus d'actualisation permanent, lié à un trait de personnalité qui permet à la personne la réalisation de son potentiel, de ses capacités et talents, ce qui permet l'accomplissement de ce qui lui semble intrinsèquement important pour elle. Pour Rogers (1973), il s'agit plutôt d'une évolution de la personne, vers plus de flexibilité (vs rigide), une vie plus active (vs 
statique), plus autonome (vs dépendante), permettant plus de créativité (vs vie prévisible) et une meilleure acceptation de soi (vs attitude de défense).

Selon Laguardia et Ryan (2000) le développement humain optimal correspond à l'eudémonie (Waterman, 1993). Cette définition du bien-être s'inspire de la conception classique d'Aristote selon laquelle les gens vivent plus ou moins en accord avec leur «vrai soi », leur daimon:

C'est le daimon qui donne l'orientation et le sens des actions d'une personne; si elle vit en accord avec son daimon, elle connaît l'eudémonie que Waterman décrit comme étant la réalisation de soi ou le fonctionnement psychologique optimal. Dans cette perspective, la réalisation de soi est possible pour quelqu'un s'il saisit les occasions de se développer et les voit comme des défis de la vie qu'il se sent capable d'affronter. Ce chercheur distingue nettement l'eudémonie et le bonheur; ce dernier ne requiert pas en lui-même de poursuivre des activités ou des buts qui stimulent la croissance personnelle et qui impliquent la réalisation de soi. Dans la poursuite du bonheur, en effet, les efforts d'un individu ne visent pas et n'atteignent pas nécessairement la réalisation de soi (Waterman, 1993, cité par Laguardia \& Ryan, 2000, p. 282)

Kasser et Ryan (2001) ajoutent que cela est notamment possible quand les personnes internalisent différents objectifs de vie et projets personnels en s'adaptant à leur environnement social (Cantor, Norem, Langston, Zirkel, Fleeson \& Cook-Flanagan, 1991 ; Little, 1989), et qu'en même temps, ces objectifs intériorisés deviennent des caractéristiques centrales de qui ils sont (Sheldon \& Kasser, 1995).

Ainsi, il apparaît clairement qu'il n'y a pas de réel consensus concernant la définition $\mathrm{du}$ fonctionnement optimal. Pour leur part, par exemple, Joseph et Wood (2010) n'établissement pas de réelles distinctions entre le fonctionnement adaptatif positif des individus, le fonctionnement humain positif ou le fonctionnement humain optimal : selon eux toutes ces expressions correspondent au fonctionnement permettant à un sujet d'être pleinement satisfait du résultat obtenu, du sentiment de maîtrise, du sentiment de contrôle... De plus, l'idée du fonctionnement optimal sous-tend aussi toute une complexité dès lors qu'il s'agira d'envisager la psychologie positive non plus au niveau individuel mais au niveau collectif et/ou sociétal.

Pour le dire autrement, par l'expression «fonctionnement humain optimal», la psychologie positive veut surtout confirmer qu'elle entend occuper un vaste champ de recherche évolutif qui concerne l'ensemble des sources de la bonne santé psychologique, et ce, dans toutes ses définitions possibles actuelles et/ou à venir. De ce fait, en fonction des cadres théories mobilisées, cela pourra éventuellement correspondre à des conceptions différentes et/ou complémentaires, comme le mettent par exemple en évidence Shankland et Martin-Krumm (2012) dans leur inventaire des outils validés en langue française permettant d'évaluer le fonctionnement optimal.

Bien entendu, le terme "optimal» fait aussi référence à l'expérience optimale (le flow) : l'émotion et le bien-être procurés par une activité en elle-même, un effet lié au sentiment de fluidité perçu au cours d'une action, lorsqu'il y a un équilibre optimal entre les exigences de la tâche et les compétences du sujet (Heutte, 2011, 2017). Conceptualisée dès 1970 par Csikszentmihalyi, cette théorie est désormais l'une des théories phares mises en lumière dans ce contexte, tout particulièrement pour ce qui concerne le développement humain et l'éducation qui sont les thèmes les plus emblématiques des travaux de Csikszentmihalyi (1975, 1990, 2014a/b). En France, comme dans toute la francophonie, la connaissance de ces travaux est hélas longtemps 
restée très confidentielle, dans la mesure où les rares ouvrages de Csikszentmihalyi traduits en français concernent des travaux qui ont plus de 20 ans (Heutte, à paraître).

\section{Être ou ne pas être positif?}

32 Le choix de l'expression "psychologie positive " pourrait laisser sous-entendre qu'il existe une psychologie négative. Bien entendu, introduire un tel jugement de valeur dans l'univers scientifique est, par principe, totalement exclu et n'aurait aucun sens. Dès le départ, Seligman et ses collègues ont très clairement répondu à certaines diatribes (notamment celle de Lazarus en 2003) qu'ils n'avaient en aucun cas l'intention de manquer de respect aux nombreux universitaires et praticiens qui ont passé la majeure partie de leur carrière à enquêter sur les états négatifs (Seligman \& Pawelski, 2003) : Seligman n'hésite pas à rappeler qu'il est l'un d'entre eux et qu'il est fier des réalisations de ce domaine. Il suggère d'ailleurs d'éviter l'usage de "psychologie négative " qu'il estime trop péjoratif, pour lui préférer celui de "psychologie comme d'habitude » (cf. psychology-as-usual, qu'il attribue à Peterson), pour décrire le travail qui se concentre sur les problèmes de l'humain.

Dans ses premières années, le mouvement de la psychologie positive s'est différencié de la psychologie comme d'habitude en mettant fortement l'accent sur le positif (pensées, émotions, traits, etc.). Dans la mesure où la psychologie comme d'habitude était axée principalement sur les états mentaux négatifs, les chercheurs impliqués dans le courant émergent de la psychologie positive pensaient que pour rétablir l'équilibre (entre le positif et le négatif) il suffisait de se concentrer sur les qualités et les résultats positifs. Indirectement, cette caractérisation induisait le message implicite que les états et les expériences ostensiblement négatifs étaient indésirables, et que les gens devraient donc viser à les éviter, alors que les qualités et les résultats positifs étaient nécessairement bénéfiques et devraient être promus et recherchés. Cependant, les critiques ont mis en évidence divers problèmes inhérents à la classification des phénomènes comme étant soit positifs soit négatifs (Held, 2004). Ainsi, au cours des dernières années, une deuxième vague (Ivtzan, Lomas, Hefferon \& Worth, 2015 ; Wong, 2011) plus nuancée de psychologie positive a germé, qui explore les complexités philosophiques et conceptuelles de l'idée même du positif, en développant une compréhension plus subtile de la nature dialectique de l'épanouissement (c'est-à-dire une interaction complexe et dynamique d'expériences positives et négatives). Dans un article récent, Lomas et Ivtzan (2016) mettent en évidence quelques études de cas concrètes concernant (1) des dichotomies saillantes (comme l'optimisme par opposition au pessimisme) qui illustrent le principe d'évaluation et (2) des processus complexes (croissance post-traumatique et amour) qui illustrent plutôt le principe de co-valence.

\section{Quelques illustrations du principe d'évaluation}

\section{L'optimisme et le pessimisme}

La première vague de la psychologie positive a été caractérisée par une tendance à valoriser l'optimisme comme partie intégrante du bien-être et à dénigrer le pessimisme en tant qu'antithèse de l'épanouissement. Cependant, comme l'a reconnu Seligman (1990), il faut faire attention à ne pas être " esclave des tyrannies de l'optimisme », mais être « capable d'utiliser le sens aigu du pessimisme de la réalité lorsque nous en avons besoin » (p. 292, traduction personnelle). Inversement, comme le résume son livre, le 
chemin du développement personnel peut impliquer des expériences qui, bien qu'elles soient ostensiblement difficiles, peuvent conduire à l'épanouissement et à la transformation. Les travaux empiriques corroborent ce point de vue et révèlent divers problèmes associés à un optimisme indu, la plupart étant liés à une sous-estimation du risque, ce qui mène à des comportements à risque (par exemple, le tabagisme (Weinstein, Marcus \& Moser, 2005)). Naturellement, en considérant les pièges de l'optimisme (le positif peut être négatif), il est possible d'inverser ce questionnement et de considérer la valeur de son pendant, le pessimisme (le négatif peut être positif). Par exemple, Norem (2001) souligne le "pouvoir positif» de la pensée négative: le pessimisme défensif (Norem \& Cantor, 1986a, 1986b) est une stratégie qui fait la médiation entre l'expectation, l'anxiété et la performance dans une situation de prise de risque. Cette stratégie implique la mise en place d'une expectation d'échec particulièrement irréaliste de façon à utiliser l'anxiété qu'elle génère pour empêcher une chute de la performance (Fenouillet, 2016). Le pessimisme défensif n'est donc pas à confondre avec une stratégie d'auto-handicap (Jones \& Berglas, 1978). En effet, contrairement aux stratégies d'auto-handicap, le pessimisme défensif n'est pas une stratégie d'échec mais de réussite. Les individus qui utilisent cette stratégie imaginent le pire afin de se motiver pour le meilleur, ils dopent leurs efforts pour empêcher l'échec.

\section{La liberté et la restriction}

La valeur de la liberté - et des concepts connexes comme l'autodétermination - est presque axiomatique au sein de la psychologie positive, considérée comme essentielle au bien-être (Ryan \& Deci, 2000). En effet, les tourments qui peuvent se produire si la liberté est refusée, comme dans l'esclavage, sont indéniables. Cependant, il a été suggéré, notamment par des penseurs existentialistes, qu'un excès de liberté, une vie sans restrictions, peut être perturbant et plutôt générer du mal-être. Kierkegaard (1834), par exemple, a estimé que le sens vertigineux des possibilités illimitées pourrait engendrer une crainte ontologique, puisque nous devons continuellement faire des choix qui façonnent irrévocablement notre vie et assumer la responsabilité des conséquences : comme le dit Sartre, les gens sont « condamnés à être libres » (1952, p. 399).

36 A l'inverse, limiter sa liberté peut être bénéfique pour le bien-être ; paradoxalement, elle peut même être libératrice. Les routines rigides de la vie monastique par exemple sont conçues pour alléger le fardeau des nombreux choix inconséquents mais incessants qui dominent la vie quotidienne (par exemple, autour de ce qu'il faut manger ou de la façon de s'habiller), libérant ainsi l'esprit pour qu'il s'engage dans l'attention "non conceptuelle et focalisée » qui est si appréciée par les méditants (Wright, 2008, p. 14). Comme l'ont montré Mischel, Shoda et Rodriguez (1989), le bienêtre dépend de la capacité à résister à des inclinaisons fugitives, à créer des stratégies pour aider à surmonter les désirs à courte vue. Ce n'est qu'ainsi que l'on peut renoncer à des satisfactions plus immédiates et poursuivre des objectifs à plus long terme comme l'observation stricte d'un régime alimentaire pour maintenir sa santé ou encore l'engagement sans aucun écart dans les études en vue d'obtenir des qualifications - qui sont finalement plus bénéfiques. Cependant, en considérant la liberté, il est de nouveau possible d'atteindre une synthèse hégélienne supérieure qui allie liberté et restriction. Cette dernière fait référence à une liberté d'attitude vitale, dans laquelle on a le 
courage d'affirmer et de poursuivre ses valeurs fondamentales: la "liberté de" (vs "liberté pour") peut encore exister dans les conditions les plus restrictives, même dans les camps de concentration en temps de guerre (Frankl, 1963).

\section{Le pardon et la colère}

37 Le pardon est généralement considéré comme bénéfique pour le bien-être de celui qui pardonne (et de celui qui est pardonné). Cependant, dans certains contextes, le pardon peut être nuisible. En effet, par exemple, les personnes qui font des attributions causales externes bienveillantes pour justifier la violence de leur partenaire (en évoquant des facteurs situationnels, comme le stress), et/ou qui pardonnent de telles transgressions, courent un plus grand risque d'être systématiquement victimes de violence (McNulty \& Fincham, 2011). Inversement, bien que la colère soit souvent présentée comme une émotion destructrice (Beck, 1999), il y a des moments où cela pourrait non seulement être une réponse plus appropriée face à un acte répréhensible que le pardon, mais qui, en fin de compte, peut servir à mieux promouvoir le bien-être à long terme. Cette réévaluation de la colère est le résultat de travaux menés par Tavris (1989), qui soutient qu'il s'agit fondamentalement d'une émotion morale, d'une réponse à une violation de l'éthique ou morale. Cela ne signifie pas non plus que la colère est toujours vertueuse ; elle peut être égoïste et/ou antisociale (Haidt, 2003). Néanmoins, comme le reconnaît Haidt, "la motivation à réparer les injustices peut aussi être fortement ressentie dans des situations de tiers, dans lesquelles le soi n'a aucun intérêt » (p. 856, traduction personnelle). Ainsi, chacun peut, et devrait sans doute, s'indigner des iniquités telles que l'oppression, et donc par exemple exiger des mesures de rétorsion ou de compensation au nom des victimes. En effet, il apparait par exemple que les grands mouvements progressistes de l'histoire récente, des droits civils au féminisme, ont été propulsés par une « colère vertueuse " qui pousse à s'engager pour un monde meilleur (Siegel, 2009). Certains travaux mettent en évidence par exemple que dans le contexte de notre société de consommation capitaliste une plus grande diversité de choix conduit souvent à des niveaux inférieurs de satisfaction subséquente à l'égard de l'élément choisi (Iyengar et Lepper, 1999). Bien que de tels troubles puissent être un luxe offert par l'abondance, ils corroborent le lien perceptif des existentialistes entre la liberté et l'anxiété.

\section{Quelques illustrations du principe de co-valence}

\section{La croissance post-traumatique}

Par exemple, la notion de trouble de stress post-traumatique (introduite dans le Manuel diagnostique et statistique des troubles mentaux (DSM-III) par l'American Psychiatric Association (1980) au lendemain de la guerre du Vietnam) était initialement clairement classée du côté négatif. Cependant, O'Leary et Ickovics (1994) ont identifié quatre réponses possibles à l'adversité: succomber (altération radicale du fonctionnement); survivre avec une déficience ; être résilient (revenir aux niveaux de fonctionnement de base d'avant l'adversité) ; et se développer (les gens se rétablissant à des niveaux de fonctionnement encore plus élevés qu'avant l'adversité). Reflétant cette dernière catégorie, Tedeschi et Calhoun (1996) ont proposé le concept de croissance post-traumatique (posttraumatic growth (PTG)), qu'ils ont défini comme «un changement positif qui se produit à la suite de la lutte contre les crises de la vie très 
difficiles » (Tedeschi et Calhoun, 2004, p. 1, traduction personnelle). Depuis lors, un grand nombre d'études ont corroboré le concept, les études ayant invariablement constaté qu'une majorité (les pourcentages varient) des personnes souffrant d'un traumatisme subissent un certain degré de PTG, le traumatisme en question allant de maladies comme le cancer (par exemple, Koutrouli, Anagnostopoulos \& Potamianos, 2012) à des catastrophes naturelles comme les cyclones (Pooley, Cohen, O'Connor \& Taylor, 2013).

\section{L'amour}

Enfin, un dernier exemple concerne l'amour. En effet, il serait certainement possible de suggérer que la question de savoir si l'amour est vécu comme positif ou négatif dépend du contexte (par exemple, amour réciproque vs non réciproque), parce que l'amour exige de mettre son destin entre les mains d'un autre, dont les actions ne peuvent être contrôlées et dont l'amour réciproque ne peut être voulu. Cependant, l'amour est en tant que tel fondamentalement dialectique; un mélange transcendant de joie et de terreur, de sécurité et de peur. C'est pour cette raison que Lomas et Ivtzan (2016) estiment que l'amour illustre tout particulièrement le "principe de co-valence " (plutôt que le "principe d'évaluation»). Car dans ce contexte, la vulnérabilité et la dysphorie potentielle sont sans doute inhérentes à l'amour: ce ne sont pas des aberrations pathologiques, mais la condition même de l'amour. Ainsi, les aspects positifs et négatifs de l'amour en sont sans doute co-créateurs. Cette perspective sur l'amour s'aligne sans doute avec la notion d'harmonisation soulignée par Delle Fave, Brdar, Freire, Vella-Brodrick et Wissing (2011) - définissable comme «l'équilibre des éléments opposés en un tout» (p.199, traduction personnelle) - qui a été évaluée comme la composante psychologique la plus importante du bonheur par les participants eux-mêmes. En tant que tel, on pourrait soutenir que les gens comprennent et apprécient intuitivement ce point que beaucoup de nos expériences les plus précieuses et les plus importantes impliquent ce genre d'équilibre dialectique, et nulle part ailleurs plus que dans le cas de l'amour (Lomas \& Ivtzan, 2016).

\section{La passion, la persistance, le flow...}

Enfin, mais cela ne peut être détaillé dans cette synthèse, dans le même ordre d'idée, nous pouvons évoquer le modèle dualistique de la passion (harmonieuse vs obsessive) développé par Vallerand (Vallerand, 2015 ; Vallerand, Blanchard, Mageau, Koestner, Ratelle, Léonard et al. 2003), ainsi que celui de la persistance (flexible vs rigide) développé avec ses collègues (Chichekian, Vallerand \& Verner-Filion, 2018). Pour notre part (Heutte, à paraître ; Heutte, Fenouillet, Martin-Krumm, \& Vallerand, 2018), nous souhaitons souligner l'amoralité du flow (Nakamura \& Csikszentmihalyi, 2002 ; 2009) qui, tout en étant toujours une expérience subjective positive pour la personne en état de flow, peut avoir des conséquences (personnelles, sociales et/ou sociétales) positives comme négatives (Dark Side of Flow).

41 Ainsi, après une jeunesse un peu trop dichotomique (Held, 2002), la psychologie positive adopte désormais une vision plus nuancée de l'idée même du positif, en développant une compréhension plus subtile du développement humain optimal. 


\section{Être ou ne pas être positiviste?}

42 standardisés spécifiques est certainement l'une des caractéristiques majeures actuelles des contributions des chercheurs à l'ensemble de la communauté scientifique. Cela permet, si cela était nécessaire, de réaffirmer que l'ancrage épistémologique de la psychologie positive s'inscrit résolument dans le champ des sciences empiriques: basées sur des méthodes de recueil de données et de traitements qui choisissent explicitement de s'exposer à la réfutation, en cohérence avec les méthodes de construction et de validation des modèles théoriques de la psychologie scientifique contemporaine, au niveau international. Le fait que les résultats de ces travaux soient régulièrement le fruit d'études comparatives à plus ou moins grande échelle fait clairement référence à l'épistémologie positiviste-empiriste de la construction scientifique, dont les fondements ont été définis au cours du XIXe siècle, notamment sous l'influence d'Auguste Comte. En effet, dans son Cours de philosophie positive, Auguste Comte a voulu donner une assise à la science positive. Quant au terme "positiviste ", il a été mis en avant plus tard, dans la seconde partie de son œuvre, à partir de 1843 et surtout 1848 (Discours sur l'ensemble du positivisme) pour désigner sa philosophie et non les sciences envers lesquelles il a manifesté une défiance croissante. Il les considérait comme des connaissances spéciales et limitées ne pouvant apporter une vue d'ensemble comme sa philosophie positiviste.

Les idées du positivisme puisent leur source dans certaines formulations de d'Alembert, Turgot, Lagrange et Condorcet. En effet, dès la seconde moitié du XVIIIe siècle, l'intention était de pouvoir expliquer le progrès de l'esprit humain (Condorcet) par le développement des "sciences positives" (mathématiques, physique, chimie, ...). En devenant positif, l'esprit renoncerait à la question "pourquoi? ", c'est-à-dire à chercher les causes premières des choses. Il se limiterait au « comment ", c'est-à-dire à la formulation des lois de la nature, exprimées en langage mathématique, en dégageant, par le moyen d'observations et d'expériences répétées, les relations constantes qui unissent les phénomènes, et permettent d'expliquer la réalité des faits. C'est sur la base de cette référence aux mathématiques que certaines sciences se pensent « exactes » et par abus de langage se définissent comme des « sciences dures». Ce que, à sa création, voudra revendiquer la psychologie, notamment avec la ferme intention de se distinguer de la philosophie (Wundt, 1913, cité par Tafreshi, Slaney \& Neufeld, 2016).

\section{Étudier objectivement la subjectivité}

Si la finalité du développement humain optimal, c'est notamment le bien-être subjectif, alors Lecomte (2012) soulève une question intéressante: est-il réellement possible d'étudier scientifiquement (c'est-à-dire en toute objectivité) la subjectivité ?

En effet, comme le rappelle Lecomte, une règle généralement prônée dans l'univers scientifique est que le chercheur, dans le cadre de son activité professionnelle, analyse des faits et se refuse à tout jugement de valeur. En d'autres termes, son travail relève de l'objectivité scientifique et non pas des croyances subjectives personnelles. «Au fil du temps, cette posture a été exprimée sous diverses formes et avec de multiples appellations, reflétant certaines nuances : distinction entre est et doit être, distinction entre jugements de faits et jugements de valeur, distinction entre le descriptif et le 
prescriptif, neutralité axiologique, critique du biais naturaliste » (Lecomte, 2012, p. 23). Ce principe trouve son origine première dans un court passage du philosophe David Hume. Dans son Traité de la nature humaine, il trouve totalement injustifié que de nombreux penseurs constatent ce qui est (is) ou n'est pas, puis en tirent des conclusions sur ce qui doit être (ought) ou non (Hume, 1739, cité par Lecomte). Au XXe siècle, le sociologue Max Weber applique cette idée de Hume à l'univers scientifique. Cependant, toujours selon Lecomte, "Weber [1965] introduit une nuance essentielle dans son propos, en distinguant le jugement de valeur (Werturteil) et le rapport aux valeurs (Wertbeziehung), ce dernier étant à ses yeux parfaitement légitime de la part d'un chercheur » (p. 23). Cette distinction permet de concilier l'étude scientifique des faits, comme des valeurs, à la condition que le chercheur soit très scrupuleux dans sa méthodologie et ses interprétations.

Concernant les effets entre les expériences personnelles du chercheur et la découverte scientifique, le point de vue du physicien devenu philosophe Mickael Polanyi est particulièrement éclairant. Dans son ouvrage Personal Knowledge, publié en 1958, Polanyi conteste l'idée d'un chercheur totalement impartial, soulignant que même dans les sciences les plus strictes (ou qui se disent les plus exactes), le savoir est toujours un art, et que l'engagement personnel et la passion sont logiquement nécessaires à la recherche. Dans cet ouvrage qui porte sur la nature et la justification du savoir scientifique, Polanyi défend, dès la préface, l'idée selon laquelle tout savoir est personnel, y compris donc, celui de la science. Ce propos conforte l'idée selon laquelle la créativité, l'intuition, voire la subjectivité du chercheur produisent des objets originaux qui relèvent d'une alchimie intime (émotionnelle, cognitive et conative). Avant d'être objectivée sous la forme d'un langage permettant sa transmission (objectivation externe), la connaissance est d'abord avant tout incarnée dans l'intimité physiologique du chercheur (objectivation interne). Il s'agit littéralement d'une

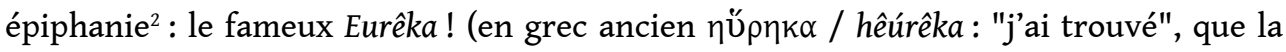
légende attribue à Archimède). Selon Csikszentmihalyi, à l'issue d'un processus créatif, trois souvenirs demeurent indissociables de l'émotion qui va marquer à tout jamais $a$ posteriori la mémoire du chercheur : "l'ardente curiosité, l'émerveillement devant un mystère sur le point d'être dévoilé, le ravissement de trouver une solution qui révèle un ordre insoupçonné» (2006, p. 10). Tous les travaux laborieux du chercheur se trouvent alors justifiés et récompensés par l'irruption soudaine d'une révélation (objectivation interne) qui peut le cas échéant effectivement produire une nouvelle connaissance, si le processus d'objectivation externe va à son terme.

\section{Interroger la quantification}

Comme les autres domaines de recherche, celui de la psychologie positive est confronté à la question de la mesure. S'il a fait du bien-être, du bonheur, du fonctionnement optimal, de la gratitude, de l'optimisme ou des institutions positives, par exemple, ses objets d'études, quels indicateurs retenir? Quels outils utiliser?

Il est de fait courant en psychologie de présumer que les phénomènes d'intérêt peuvent et doivent être représentés par des nombres, et que les inférences doivent être basées sur l'analyse mathématique de ces représentations numériques. Dans leur article paru en 2016, Tafreshi, Slaney et Neufeld se sont interrogés sur la prédominance de la quantification dans la pratique de la recherche en psychologie. Pour y parvenir, ils ont 
tout d'abord passé en revue plusieurs arguments critiques avancés par des théoriciens de la psychologie, ainsi que par des philosophes et des historiens de la psychologie.

\section{Une brève histoire des fondements de la quantification en psychologie moderne}

Tafreshi et ses collègues rappellent que les fondements de la quantification en psychologie sont généralement attribués aux travaux de Gustav Fechner à la fin du XIXe siècle en Allemagne. Dans son ouvrage Elemente der Psychophysik, Fechner (1860) plaidait pour une psychologie (ou psychophysique) basée sur des fondements mathématiques en établissant des comparaisons entre l'étude de la psychologie et de la physique. L'attrait de la quantification a également balayé la Grande-Bretagne à cette époque, notamment via la création de la revue Biometrika fondée en 1901 par Francis Galton et Karl Pearson. En 1946, le psychologue américain, Stevens publie On the Theory of Scales of Measurement qui est sans doute l'ouvrage le plus important dans le domaine de la mesure psychologique au XXe siècle. Il définit la mesure comme «l'attribution de chiffres à des objets ou à des événements selon des règles " (Stevens, 1946, p. 677, traduction personnelle). En proposant une définition aussi souple de la mesure, Stevens a laissé entendre que tout phénomène pouvait être considéré comme mesurable, à condition qu'un ensemble de règles permettant aux chercheurs d'attribuer des valeurs numériques à des instances spécifiques du phénomène soient explicitement définis (Michell, 2004; Trendler, 2009). Très rapidement, l'utilisation de tests standardisés pour évaluer les capacités intellectuelles et mentales est devenue un point de mire prédominant des psychologues américains. Charles Spearman a été l'un des principaux contributeurs au développement des tests psychologiques au début du XXe siècle. Spearman (1904a/b) a adopté la méthode Galton-Pearson de l'analyse corrélationnelle comme moyen d'étudier les relations entre les mesures et, sur la base de ces études corrélationnelles, il a développé la méthode psychométrique communément appelée aujourd'hui analyse factorielle.

\section{La quantification : un habitus implicite}

En se basant ainsi sur l'histoire de la quantification en psychologie moderne, Tafreshi et ses collègues (2016) soutiennent que la quantification en psychologie a toujours été, et continue d'être, une pratique généralement non réfléchie. Citant comment Wundt, dans son article de 1913 intitulé Psychology's Struggle for Existence [la lutte de la psychologie pour son existence] craignait qu'une séparation entre la psychologie et la philosophie ne conduise à un manque de pensée critique, ils estiment que cette séparation a effectivement été préjudiciable à la psychologie. Car le manque de réflexion philosophique dans le domaine a contribué à l'indifférence de la psychologie à l'égard d'importantes questions conceptuelles et méthodologiques. De plus, le manque d'intérêt de la psychologie à l'égard de l'histoire des pratiques de quantification dans la discipline a entraîné un manque de réflexion critique sur les motifs qui motivent son utilisation. Bien que le positivisme soit le plus souvent associé à la quantification et aux méthodes quantitatives, il n'est pas clair que ce couplage soit totalement justifié (Comte, 1907). Citant Hacking (1983) et Slaney (2001), Tafreshi et ses collègues suggèrent que la psychologie moderne est principalement fondée sur une forme de réalisme plutôt que sur le positivisme. Nous retrouvons ici l'idée force de Kuhn (1972) selon laquelle, contrairement à ce qu'énonce Popper, un paradigme scientifique n'est pas rejeté dès qu'il est réfuté, mais seulement quand il peut être remplacé. Kuhn 
rappelle aussi que les raisons qui prévalent à un changement de paradigme ne sont que partiellement rationnelles, car les opinions et choix des scientifiques sont selon lui tributaires de leurs expériences, de leurs croyances et de leurs visions du monde.

\section{La carte n'est pas le territoire : plaidoyer pour approche méthodologique plus équilibrée}

51 Selon Vautier (2015), l'usage générique du terme "évaluation" possède par ailleurs une ambiguïté descriptive et appréciative redoutable : dans le champ de la psychologie, le calcul d'un score n'est ni un jugement de valeur, ni encore moins en tant que tel le résultat d'un mesurage (comme il serait possible de le faire pour la taille, le poids ou l'âge d'un enfant, par exemple). Cela implique que l'utilisation du terme de mesure est trompeuse puisque la finalité de ces outils, c'est de fournir « les moyens d'exprimer ces observations [les réponses] sous une forme telle que soient possibles la comparaison [des] individus entre eux et la comparaison de chacun avec les "normes" (descriptives) de la population à laquelle ils appartiennent» (Reuchlin, 1969, p. 22). Ces outils ont surtout pour vocation de permettre de mieux manipuler des constructions théoriques, notamment de fournir le moyen de vérifier la plausibilité de modélisation, ou d'hypothèses.

Pour notre part, totalement conscient de ces limites à l'objectivité, nous considérons que dans l'adolescence de la psychologie positive, dans le souci de se démarquer de la psychologie humaniste et aussi du fait des habitus culturels de la recherche nord américaine en psychologie (très marquée par l'empirisme de la recherche médicale), il peut être compréhensible que certains excès de jeunesse aient été commis. Cependant, il faut savoir que progressivement de nombreux chercheurs "non nord américains", notamment en Europe, souhaitent s'émanciper de l'emprise de la culture des fondateurs de la psychologie positive. Il est ainsi intéressant de noter que sur le portail de l'European Network for Positive Psychology (ENPP), il est clairement indiqué que l'utilisation de divers modèles de recherche épistémologique qualitative (Hefferon, Ashfield, Waters \& Synard, 2017) est plus largement acceptée en Europe (qu'en Amérique du Nord), et cette tendance est de plus en plus accessible à travers le monde. En partie sous l'influence de certains chercheurs européens (e.g. Delle Fave, 2013 ; Delle Fave, Massimini, et Bassi, 2011; Knoop et Delle Fave, 2012; Leontiev, 2012...), de nombreuses initiatives de la communauté des chercheurs français et francophones s'inscrivent dans ces perspectives, notamment en revendiquant une conception plus sociale et moins individualiste de l'individu (e.g. Csillik, 2017 ; Fenouillet, 2016 ; Gendron, 2016, 2018 ; Heutte, 2011, 2019 ; Lecomte, 2014 ; Martin-Krumm, \& Tarquinio, 2011, 2019; Masson, 2018; Shankland, 2019; Vallerand, 2015...). De tous ces éléments, il ressort qu'après l'apparition d'une deuxième vague (Ivtzan et al., 2015; Wong, 2011) plus nuancée de la psychologie positive (qui explore les complexités philosophiques et conceptuelles de l'idée même du positif et du négatif, en développant une compréhension plus subtile de la nature dialectique de l'épanouissement), une approche méthodologique plus équilibrée (quantitatif vs qualitatif), ainsi qu'un regard critique concernant la "zone d'ombre" (Phipps, 2011) ${ }^{3}$ et l'éthique de certains engagements personnels de chercheurs emblématiques fondateurs de la psychologie positive aux États Unis (Hoffman, Carter, Lopez, Benzmiller, Guo, Latifi \& Craig, 2015), en constituera peut-être la troisième vague. 
Quoi qu'il en soit, il fallait certainement nécessairement commencer par baliser, puis cartographier le paysage de la psychologie positive, notamment chercher à identifier la consistance des concepts à étudier et tenter de définir la plausibilité de lois générales, dans la mesure du possible prédictives, afin de pouvoir interroger certaines évidences : "Guba et Lincoln [1998] définissent ce qu'ils dénomment la "vérité4" (vérité avec des guillemets) comme la représentation la plus informée et sophistiquée faisant consensus » (Avenier, 2011, p. 376). Cependant, reprenant à notre compte la métaphore de Korzybski (1933), nous sommes totalement conscients qu'une carte n'est pas le territoire. En effet, les modèles théoriques sont des constructions élaborées par des chercheurs lorsqu'ils essaient de donner du sens à l'interprétation des résultats de leurs expériences. Soumis via une autorité procédurale (notamment pour publications dans des revues scientifiques), ces modèles théoriques deviennent des "réalités socialement construites» (Guba et Lincoln, 1989, p. 86, cités par Avenier, 2011, p. 378) qui relèvent du niveau ontologique, c'est-à-dire de ce que Korzybski désigne par « territoire ». Comme suggère Cosinschi (2008), il est possible d'étendre les propositions de Korzybski, pour les mettre en corrélation dans un champ logique ternaire, qui est le suivant :

- la carte n'est pas le territoire $\rightarrow$ un modèle théorique est nécessairement une simplification/ réduction du réel, il a pour objet de tenter de mettre en évidence un nombre restreint de variables (principe du rasoir d'Occam);

- la carte ne représente pas tout le territoire $\rightarrow$ aucun modèle théorique ne peut expliquer tous les comportements humains;

- la carte est auto-réflexive $\rightarrow$ la plus grande finalité pratique/pragmatique d'un modèle théorique est de faciliter l'étude d'un processus psychologique qui par nature est nécessairement complexe.

Enfin, en guise de conclusion provisoire, comme un pied de nez à la fois positif et positiviste, nous terminons par cette citation de Victor Brochard: "L'homme n'est capable de science que parce qu'il est libre; c'est aussi, parce qu'il est libre, qu'il est sujet à l'erreur. » (1897, p. 7), en ajoutant pour notre part : jusqu'à preuve du contraire.

In fine, nous estimons que la levée de certaines ambigüités et/ou incompréhensions, permet, si cela était nécessaire, de réaffirmer que l'encrage épistémologique de la psychologie positive s'inscrit résolument dans le champ des sciences empiriques: basées sur des méthodes de recueil de données et de traitements qui choisissent explicitement de s'exposer à la réfutation, en cohérence avec les méthodes issues de la psychologie scientifique contemporaine, dont les perspectives européennes souhaitent s'émanciper progressivement de l'emprise de la culture de ses fondateurs. Cela doit permettre de clairement distinguer la psychologie positive des niaiseries de la vulgate des vendeurs de "trucs et astuces du bonheur" qui surfent avec intérêt sur la médiatisation excessive de la marchandisation du bien-être et de la crédulité naïve de ceux qui y sont un peu trop réceptifs. 


\section{BIBLIOGRAPHIE}

Avenier, M.J. (1997), La stratégie chemin faisant, Economica.

Avenier, M. J. (2011). Les paradigmes épistémologiques constructivistes : post-modernisme ou pragmatisme? Management \& avenir, (3), 372-391.

Beck, A. T. (1999). Prisoners of Hate : The Cognitive Basis of Anger, Hostility, and Violence. New York : Harper Collins.

Brochard, V. (1897). De l'erreur. Félix Alcan.

Brown, N. J., Lomas, T., \& Eiroa-Orosa, F. J. (Eds.). (2017). The Routledge international handbook of critical positive psychology. Routledge.

Cantor, N., Norem, J., Langston, C., Zirkel, S., Fleeson, W., \& Cook-Flannagan, C. (1991). Life tasks and daily life experience. Journal of Personality, 59(3), 425-451.

Chichekian, T., Vallerand, R.J., \& Verner-Filion, J. (2018). Passion and persistence : A look at adaptive and less adaptive consequences. Manuscrit soumis.

Comte, A. (1907). Discours sur l'ensemble du positivisme. Société positiviste internationale.

Cosinschi, M. (2008). Alfred Korzybski et la pragmatique de la carte. Analele stiintifice ale Universitatii" Alexandru Ioan Cuza" din Iasi-seria Geografie, 54, 11-18.

Csikszentmihalyi, M. (1975). Beyond boredom and anxiety. Jossey-Bass San Francisco.

Csikszentmihalyi, M. (1990). Flow: The Psychology of Optimal Experience. New York, Harper and Row.

Csikszentmihalyi, M. (2006). La créativité : psychologie de la découverte et de l'invention. R. Laffont.

Csikszentmihalyi, M. (2014a). Flow and the foundations of positive psychology. Springer, Dordrecht : Netherlands.

Csikszentmihalyi, M. (2014b). Applications of Flow in Human Development and Education. Springer, Dordrecht : Netherlands

Csillik, A. (2017). Les ressources psychologiques : Apports de la psychologie positive. Dunod, Paris, France.

Delle Fave, A. (2013). La psychologie positive entre idéal et réalité. $1^{\mathrm{er}}$ Congrès francophone de psychologie positive. Metz, France 21-22 novembre 2013

Delle Fave, A., Brdar, I., Freire, T., Vella-Brodrick, D., \& Wissing, M. (2011). The eudaimonic and hedonic components of happiness : Qualitative and quantitative findings. Social Indicators Research, 100(2), 185-207.

Delle Fave, A., Massimini, F., et Bassi, M. (2011). Psychological selection and optimal experience across cultures: Social empowerment through personal growth. Springer Science et Business Media.

Eidelson, R., Pilisuk, M., \& Soldz, S. (2011). The dark side of comprehensive soldier fitness. American Psychologist, 66 (7), 643-644.

Fechner, G. T. (1860/1966). Elemente der psychophysik. Leipzig : Breitkopf \& Hartel. English translation by H.E. Adler, Elements of psychophysics, (Vol. 1). D.H. Howes \& E.G. Boring (Eds). New York, NY : Holt, Rinehart \& Winston

Fenouillet, F. (2016). Les théories de la motivation. Dunod, Paris : France. 
Frankl, V. E. (1963). Man's Search for Meaning : An Introduction to Logotherapy. New York : Washington Square Press.

Froh, J. J. (2004). The history of positive psychology : Truth be told. NYS psychologist, 16(3), 18-20. Gendron, B. (2016). Management des risques psycho-sociaux et capital émotionnel pour une qualité de vie au travail et vers des organisations capacitantes : essai réflexif et intervention sur le stress dans le monde du soin et de l'éducation via l'approche de la pleine conscience, de l'acceptation et de l'engagement et de management de projet. (Thèse de doctorat, université Paul Valéry-Montpellier). En ligne : https:// tel.archives-ouvertes.fr/tel-01486114/document

Gendron, B. (2018). The power of the Emotional Capital in Education: Executives functions and Medidation/Mindfulness. Publibook/Société écrivains

Haidt, J. (2003). The moral emotions. In R. J. Davidson, K. R. Scherer \& H. H. Goldsmith (Eds.), Handbook of affective sciences (pp. 852-870). Oxford : Oxford University Press.

Held, B. S. (2002). The tyranny of the positive attitude in America : Observation and speculation. Journal of Clinical Psychology, 58(9), 965-991.

Held, B.S. (2004). The negative side of positive psychology. Journal of Humanistic Psychology, 44(1), 9-46.

Hefferon, K., Ashfield, A., Waters, L. \& Synard, J. (2017). Understanding optimal human functioning-The 'call for qual'in exploring human flourishing and well-being. The Journal of Positive Psychology, 12(3), 211-219.

Heidegger, M. (1927/1986). Être et temps. Paris, France : Gallimard.

Heutte, J. (2011). La part du collectif dans la motivation et son impact sur le bien-être comme médiateur de la réussite des étudiants : complémentarités et contributions entre l'autodétermination, l'auto-efficacité et l'autotélisme (thèse de doctorat, Université Paris Ouest-Nanterre-La Défense, Nanterre). https:// tel.archives-ouvertes.fr/tel-00933690/

Heutte, J. (2017). Motivations, volition et expérience du flow : quelques références théoriques pour l'étude des communautés d'apprenance. Dans O. Las Vergnas (dir.), Le e-learning informel ? Des apprentissages diffus, noyés dans la participation en ligne (p. 199-214). Paris, France : Archives contemporaines.

Heutte, J. (2019). Les fondements de l'éducation positive : perspective psychosociale et systémique de l'apprentissage. Dunod, Paris : France.

Heutte, J. (à paraître). Psychologie du Flow : science de l'expérience optimale. Dunod, Paris : France.

Heutte, J., Fenouillet, F., Martin-Krumm, C. \& Vallerand, R. J. (2018). Mediating effects of Flow on harmonious passion: contribution to the exploration of bright side and dark side of Flow in educational context. 9 $^{\text {th }}$ European Conference on Positive Psychology (ECPP 2018). Budapest, Hongrie.

Hoffman, D. H., Carter, D. J., Lopez, C. R. V., Benzmiller, H. L., Guo, A. X., Latifi, S. Y., \& Craig, D. C. (2015). Independent review relating to APA ethics guidelines, national security interrogations, and torture. Sidley Austin LLP.

Husserl, E. (1913/1950). Idées directrices pour une phénoménologie et une philosophie phénoménologique pures (Tome Premier). Paris, France : Gallimard,[1913].

Ivtzan, I., Lomas, T., Hefferon, K., \& Worth, P. (2015). Second wave positive psychology : Embracing the dark side of life. London, Royaume-Uni : Routledge. 
Iyengar, S. S., \& Lepper, M. R. (1999). Rethinking the value of choice : A cultural perspective on intrinsic motivation. Journal of Personality and Social Psychology, 76(3), 349-366.

James, W. (1890). The principles of psychology (Vols. 1 \& 2). New York : Holt.

Jones, E. E., \& Berglas, S. (1978). Control of attributions about the self through self-handicapping strategies : The appeal of alcohol and the role of underachievement. Personality and Social Psychology Bulletin, 4(2), 200-206.

Joseph, S., \& Wood, A. (2010). Assessment of positive functioning in clinical psychology : Theoretical and practical issues. Clinical Psychology Review, 30, 830-838.

Kasser, T. \& Ryan, R. M. (2001). Be careful what you wish for : Optimal functioning and the relative attainment of intrinsic and extrinsic goals. Dans P. Schmuck \& K. M. Sheldon, Life goals and well-being : Towards a positive psychology of human striving (p. 116-131). Ashland, OH, : Hogrefe \& Huber Publishers.

Kierkegaard, S. (1843/2000). Crainte et tremblement. Paris, France : Payot.

Kruger, J. \& Dunning, D. (1999). Unskilled and unaware of it : How difficulties in recognizing one's own incompetence lead to inflated self-assessments. Journal of personality and social psychology, $77(6), 1121$.

Knoop, H.H., et Delle Fave, A. (eds.) (2012). Well-being and cultures : Perspectives from positive psychology (vol. 3). Springer Science et Business Media.

Korzybski, A. (1933). Science and Sanity: An Introduction to Non Aristotelian Systems and General Semantics, traduction française. 2007, Une carte n'est pas le territoire, Editions de l'Eclat, Paris. Koutrouli, N., Anagnostopoulos, F., \& Potamianos, G. (2012). Posttraumatic stress disorder and posttraumatic growth in breast cancer patients : a systematic review. Women \& Health, 52(5), 503-516.

Kuhn, T. S. (1972). La structure des révolutions scientifiques. Paris, Flammarion.

Laguardia, J. G., \& Ryan, R. M. (2000). Buts personnels, besoins psychologiques fondamentaux et bien-être : théorie de l'autodétermination et applications. Revue québécoise de psychologie, 21(2), 281-304.

Lazarus, R. S. (2003). Does the positive psychology movement have legs? Psychological Inquiry, 14(2), 93-109.

Lecomte, J. (2002). Qu'est-ce que la résilience ? Question faussement simple. Réponse nécessairement complexe. Pratiques psychologiques, (1), 7-14.

Lecomte, J. (2012). Est-il justifié de parler de psychologie positive ? Les cahiers internationaux de psychologie sociale, (1), 21-36.

Lecomte, J. (2014). Introduction à la Psychologie Positive, Editions Dunod.

Leontiev, D. (2012). Motivation, consciousness, and self-regulation. New York, NY: Nova Science Publishers

Little, B. R. (1989). Personal project analysis : Trivial pursuits, magnificent obsessions, and the search for coherence. In D. M. Buss, \& N. Cantor (Eds.), Personality psychology : Recent trends and emerging issues. New York : Springler Verlag. (pp. 15-31).

Lomas, T., \& Ivtzan, I. (2016). Second wave positive psychology : exploring the positive-negative dialectics of wellbeing. Journal of Happiness Studies, 17(4), 1753-1768. 
Martin-Krumm, C. \& Tarquinio, C. (2011). Traité de psychologie positive. Bruxelles, Belgique : De Boeck.

Martin-Krumm, C. \& Tarquinio, C. (2019). Psychologie positive : Etat des savoirs, champs d'application et perspectives. Dunod, Paris, France.

Maslow, A. H. (1954). Personality and motivation. Harlow, England : Longman.

Maslow, A. H. (1968). Toward a psychology of being. New York : Van Nostrand Reinhold.

Masson, J. (2018). Bienveillance et réussite scolaire. Dunod, Paris, France.

McNulty, J. K., \& Fincham, F. D. (2011). Beyond positive psychology ? Toward a contextual view of psychological processes and well-being. American Psychologist, 67(2), 101-110.

Michell, J. (2000). Normal science, pathological science, and psychometrics. Theory and Psychology, 10(5), 639-667

Mischel, W., Shoda, Y., \& Rodriguez, M. I. (1989). Delay of gratification in children. Science, 244(4907), 933-938.

Myers, D. G. (2000). The funds, friends, and faith of happy people. American psychologist, 55(1), 56.

Nakamura, J., \& Csikszentmihalyi, M. (2002). The concept of flow. Handbook of positive psychology, 89-105.

Nakamura, J., \& Csikszentmihalyi, M. (2009). Flow Theory and Research. Oxford Handbook of Positive Psychology, 195.

Norem, J. K. (2001). The Positive Power of Negative Thinking. New York : Basic Books.

Norem, J. K., \& Cantor, N. (1986a). Anticipatory and post hoc cushioning strategies : Optimism and defensive pessimism in "risky" situations. Cognitive Therapy and Research, 10, 347-362.

Norem, J. K., \& Cantor, N. (1986b). Defensive pessimism : Harnessing anxiety as motivation. Journal of Personality and Social Psychology, 51, 1208-1217.

O'Leary, V. E., \& Ickovics, J. R. (1994). Resilience and thriving in response to challenge : an opportunity for a paradigm shift in women's health. Women's health (Hillsdale, NJ), 1(2), 121-142

Peirce, C. S. (1877). The fixation of belief. Popular Science Monthly 12, 1-15.

Peirce, C. S. \& Hetzel, A. (1878). How to make our ideas clear. Popular Science Monthly 12, 286-302.

Phipps, S. (2011). Positive psychology and war : An oxymoron. American Psychologist, 66 (7), 641-642.

Polanyi, M. (1958). Personal Knowledge. Routledge, London : UK.

Pooley, J. A., Cohen, L., O'Connor, M., \& Taylor, M. (2013). Posttraumatic stress and posttraumatic growth and their relationship to coping and self-efficacy in Northwest Australian cyclone communities. Psychological Trauma: Theory, Research, Practice, and Policy, 5(4), 392-399.

Pressey, S. L. (1927). A machine for automatic teaching of drill material. School \& Society. Rathunde, K. (2001). Toward a psychology of optimal human functioning: What positive psychology can learn from the "experiential turns" of James, Dewey, and Maslow. Journal of Humanistic Psychology, 41(1), 135-153.

Reuchlin, M. (1969). Les méthodes en psychologie. Presses universitaires de France.

Rogers, C. R. (1973). My philosophy of interpersonal relationships and how it grew. Journal of Humanistic Psychology, 13(2), 3-15. 
Ryan, R. M., \& Deci, E. L. (2000). Self-determination theory and the facilitation of intrinsic motivation, social development, and well-being. American psychologist, 55(1), 68-78.

Ryan, R. M., \& La Guardia, J. G. (2000). What is being optimized over development ? A selfdetermination theory perspective on basic psychological needs across the life span. Dialogues on psychology and aging, 145-172.

Sartre, J.-P. (1943). L'être et le néant. Essai d'ontologie phénoménologique. Paris, France : Gallimard

Sartre, J. P. (1952). Saint Genet : comédien et martyr. Gallimard.

Seligman, M. E. P. (1990). Learned optimism. New York : Random House.

Seligman, M. E. P., \& Csikszentmihalyi, M. (2000). Positive psychology : An introduction. American Psychologist, 55(1), 5-14.

Seligman, M. E. P. \& Pawelski, J. O. (2003). Positive psychology : FAQS. Psychological Inquiry, 14(2), 159-163.

Shankland, R. \& Martin-Krumm, C. (2012). Évaluer le fonctionnement optimal : échelles de psychologie positive validées en langue française. Pratiques psychologiques, 18(2), 171-187.

Shaffer, J.B.P. (1978). Humanistic psychology. Englewood Cliffs, NJ : Prentice-Hall, Inc.

Sheldon, K. M., \& Kasser, T. (1995). Coherence and congruence : Two aspects of personality integration. Journal of personality and social psychology, 68(3), 531.

Siegel, A. (2009). Justice Stevens and the Seattle schools case : A case study on the role of righteous anger in constitutional discourse. UC Davis Law Review, 43, 927-937.

Skinner, B. F. (1958). Teaching machines. Science, 128(3330), 969-977.

Skinner, B. F. (1971). Beyond freedom and dignity. New York, NY : Alfred A. Knopf.

Skinner, B. F. (1981). Innovation in science teaching. Science, 212, 283.

Skinner, B. F. (1984). The shame of American education. American Psychologist, 39(9), 947-954.

Spearman, C. (1904a). The proof and measurement of association between two things. The American journal of psychology, 15(1), 72-101.

Spearman, C. (1904b). "General Intelligence" objectively determined and measured. The American Journal of Psychology, 15(2), 201-292.

Stevens, S. S. (1946). On the theory of scales of measurement. Science, 103(2684), 67-680.

Sutich, A. J., \& Vich, M. A. (1969). Readings in humanistic psychology. Free Press.

Tafreshi, D., Slaney, K. L., \& Neufeld, S. D. (2016). Quantification in psychology : Critical analysis of an unreflective practice. Journal of Theoretical and Philosophical Psychology, 36(4), 233.

Tavris, C. (1989). Anger : The Misunderstood Emotion. New York : Touchstone.

Taylor, E. (2001). Positive psychology and humanistic psychology : A reply to Seligman. Journal of Humanistic Psychology, 41(1), 13-29.

Tedeschi, R., \& Calhoun, L. (1996). The Posttraumatic Growth Inventory : Measuring the positive legacy of trauma. Journal of Traumatic Stress, 9(3), 455-471.

Tedeschi, R. G., \& Calhoun, L. G. (2004). Posttraumatic growth : Conceptual foundations and empirical evidence. Psychological Inquiry, 15(1), 1-18. 
Trendler G. (2009). Measurement theory, psychology and the revolution that cannot happen. Theory and Psychology, 19, 579-599

Vallerand, R. J. (2015). The psychology of passion : A dualistic model. Oxford University Press, New York, NY.

Vallerand, R. J., Blanchard, C., Mageau, G. A., Koestner, R., Ratelle, C., Léonard, M., Gané, M. \& Marsolais, J. (2003). Les passions de l'âme : on obsessive and harmonious passion. Journal of personality and social psychology, 85(4), 756.

Vautier, S. (2015). La psychotechnique des aptitudes. Pour différencier une sociotechnique de l'évaluation sans mesurage et une psychologie balbutiante de la compréhension de la performance. Pratiques psychologiques, 21(1), 1-18.

Waterman, A. S. (1984). Identity formation : Discovery or creation ?. The Journal of Early Adolescence, 4(4), 329-341.

Waterman, A. S. (1993). Laga Two conceptions of happiness : Contrasts of personal expressiveness (eudaimonia) and hedonic enjoyment. Journal of Personality and Social Psychology, 64(4), 678-691.

Weinstein, N. D., Marcus, S. E., \& Moser, R. P. (2005). Smokers' unrealistic optimism about their risk. Tobacco control, 14(1), 55-59.

Wright, D. S. (2008). Introduction : Rethinking ritual practice in Zen Buddhism. In S. Heine \& D. S. Wright (Eds.), Zen Ritual : Studies of Zen Buddhist Theory in Practice (pp. 3-20). Oxford : Oxford University Press.

Wong, P. T. P. (2011). Positive psychology 2.0 : Towards a balanced interactive model of the good life. Canadian Psychology/Psychologie Canadienne, 52(2), 69

\section{NOTES}

1. Effet "Dunning-Kruger". La variation de la confiance dans ses capacités en fonction de l'expérience est le résultat de biais cognitifs qui conduisent les personnes les moins compétentes à surestimer leurs capacités (parce qu'elles sont dans l'ignorance de leur ignorance) et les plus compétentes à les sous-estimer (parce qu'elles surestiment la complexité de ce qu'elles doivent maîtriser). Dans le cas des réseaux sociaux, cela peut notamment expliquer pourquoi les personnes ignorantes sont plus à même de croire ce qui est transmis par quelqu'un qui n'a aucun doute sur ce qu'il affirme, même quand cela pourrait être contredit par un expert qui sera généralement plus nuancé dans ses propos, donc souvent plus difficile à comprendre (Kruger \& Dunning, 1999).

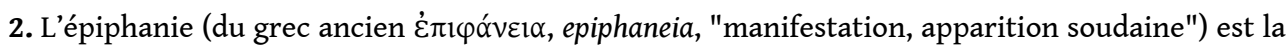
compréhension soudaine de l'essence ou de la signification de quelque chose (selon le dictionnaire Larousse).

3. Le cas échéant, ne pas hésiter à consulter certains articles publiés dans American Psychologist, en octobre 2011, soit 10 mois après le $n^{\circ}$ spécial Comprehensive Soldier Fitness coordonné par Seligman. Dans lesquels certains collègues nord américains (notamment Eidelson, Pilisuk, \& Soldz, 2011; Phipps, 2011) évoquent le désarroi, puis la colère, et enfin un sentiment de honte pour la profession, concernant l'engagement personnel de certains de leurs collègues dans ce programme financé par l'US Army.

4. Selon Guba et Lincoln (1989, cités par Avenier, 2011), le paradigme épistémologique constructiviste comporte une hypothèse fondatrice d'ordre ontologique. Celle-ci pose qu'il 
n'existe pas de réalité objective, mais de multiples réalités socialement construites, et que cellesci ne sont pas gouvernées par des lois naturelles, causales ou d'autre sorte.

\section{RÉSUMÉS}

Sous l'impulsion initiale de Seligman et de Csikszentmihalyi, le début de ce millénaire restera marqué par l'émergence de la psychologie positive qui peut être considérée comme la quatrième vague dans l'évolution de la psychologie, les trois premières vagues étant, respectivement, le modèle de la maladie, le behaviorisme et la psychologie humaniste. Depuis, progressivement, sur tous les continents, le monde de la psychologie et de la psychothérapie amorce une évolution radicale. Cependant dans la communauté française et francophone ce champ de recherche semble porteur de nombreux malentendus. Afin de lever certains doutes, cet article souhaite rappeler les fondements épistémologiques (historique, philosophique, méthodologique...) de la science de l'expérience optimale en tant que champ de recherche empirique concernant l'expérience positive subjective, depuis la création du Positive Psychology Steering Commitee constitué par Csikszentmihalyi, Diener, Hall Jamieson, Peterson et Vaillant, en 1999, jusque ses dernières évolutions, notamment sous l'influence de la communauté des chercheurs européens. Il a aussi pour objectif de lever quelques ambiguïtés originelles concernant certains termes (optimal, positif et positiviste) qui parfois peuvent être l'objet de controverses (voire de polémiques idéologiques infondées) quand ils sont mal traduits ou interprétés.

Under the Seligman and Csikszentmihalyi's seminal work, the new millennium is characterized by the emergence of positive psychology, which can be considered as the fourth wave in the evolution of psychology, the first three waves being, respectively, the disease model, behaviourism and humanist psychology. Since then all over the world, believers of psychology and psychotherapy have undertaken a radical turn. However, in the French and Francophone community, this field of research seems to be the source of many misunderstandings. In order to clarify certain doubts, this paper aims to summarise the epistemological foundations (historical, philosophical, methodological...) of the science of optimal experience as a field of empirical research regarding subjective positive experience, since the creation of the Positive Psychology Steering Committee constituted by Csikszentmihalyi, Diener, Hall Jamieson, Peterson and Vaillant in 1999, until its last developments, particularly under the influence of European researchers. It also aims to remove some original ambiguities regarding certain terms (optimal, positive and positivist) which can sometimes be the subject of controversy (or even polemics) when they are mistranslated or interpreted.

\section{INDEX}

Mots-clés : Psychologie positive, développement humain optimal, positivisme, épistémologie

Keywords : Positive psychology, optimal human development, positivism, epistemology 
AUTEUR

JEAN HEUTTE

Université de Lille, EA 4354 - CIREL - Centre Interuniversitaire de Recherche en Education de Lille 